\title{
Hepatitis C testing trends among large commercially insured populations, 2011-2017
}

\author{
Mohammed A. Khan 2, 1, Jae Eui Soh ${ }^{2,1}$, William W. Thompson ${ }^{2}$, Noele P. Nelson ${ }^{2}$ \\ ${ }^{1}$ Emory University, Atlanta, Georgia, United States, ${ }^{2}$ Centers for Disease Control and Prevention, Atlanta, Georgia, United States \\ Objective
}

We estimated the rate of hepatitis C testing between 2011 and 2017 among persons with commercial health insurance coverage and compared rates by birth cohort.

\section{Introduction}

Hepatitis $\mathrm{C}$ virus (HCV) infection is the most common blood-borne infection in the US, and a leading cause of liver-related morbidity and mortality. Approximately 3.5 million individuals in the US were estimated to have been living with hepatitis $\mathrm{C}$ in 2010, and approximately half of them were unaware that they were infected. Among HCV infected individuals, those born between 1945 and 1965 (usually referred to as the baby boomer cohort) represent approximately 75\% of current cases. Because of the substantial burden of disease among this age group, CDC expanded its existing hepatitis C risk-based testing recommendations to include a one-time HCV antibody test for all persons born between 1945 and 1965. The United States Preventive Services Task Force (USPSTF) subsequently made the same recommendation in June 2013.

\section{Description}

We obtained data from the 2011-2017 IBM MarketScan® Commercial Claims and Encounters and Medicare Supplemental and Coordination of Benefits databases. These data consist of inpatient and outpatient service claims for persons with employersponsored health insurance coverage and their dependents. This analysis was restricted to adults 18 years of age and older with continuous enrollment in a commercial or Medicare Supplemental plan for at least one calendar year during the study period (a 45day gap in coverage was allowed) who received outpatient prescription drug claims data feeds. Claims for hepatitis $\mathrm{C}$ antibody testing were identified using Current Procedural Terminology (CPT) codes (80074, 86803). We defined the annual hepatitis C testing rate as the number of patients with an HCV antibody test claim divided by the total number of study-eligible enrollees in a given calendar year. Testing rates were calculated for persons born between 1945 and 1965 and all other adults.

There were 54,298,561 unique adults who were continuously enrolled for at least one calendar year during the study period. Among these, 4,629,040 (9\%) had one or more inpatient or outpatient service claim with a CPT code for hepatitis C antibody testing during the study period. The overall estimated annual testing rate increased from $2.2 \%$ in 2011 to $5.3 \%$ in 2017 . The testing rate increased from $1.7 \%$ to $7.8 \%$ among the $1945-1965$ birth cohort and $2.5 \%$ to $4.0 \%$ in other birth cohorts. The average annual percent change in testing was $30.1 \%$ among the 1945-1965 birth cohort and $8.2 \%$ among other birth cohorts. Testing rate increased markedly (64.1\%) between 2016 and 2017 in the 1945-1965 birth cohort, but not in other birth cohorts (7.7\%).

In this sample of individuals covered by commercial insurance, hepatitis $\mathrm{C}$ testing rates have increased slowly between 2011 and 2016. In 2017, there was a substantial increase in testing rates among the Baby Boomer cohort due most likely to an increase in awareness of CDC and USPSTF recommendations by both providers and individual patients associated with CDC health promotion efforts and increased marketing efforts by drug manufacturers. Efforts should continue to promote and increase the awareness of these recommendations and have people tested and treated for $\mathrm{HCV}$. 